\title{
Paramedian Approach to Coccygectomy in the Treatment of Chronic Coccydynia: A Technical Note
}

\author{
Kyle Geiger ${ }^{1}$, Carson Fuller ${ }^{1}$, Crystal Gomez ${ }^{2}$, Miguel A. Schmitz ${ }^{1,2}$ \\ Department of Orthopaedics, ${ }^{1}$ Washington State University, Elson S. Floyd College of Medicine, Spokane, WA, ${ }^{2}$ Alpine Orthopaedic and \\ Spine, Spokane, WA, U.S.A.
}

Corresponding Author:

Kyle Geiger, BS

Department of Orthopaedics,

Washington State University, Elson

S. Floyd College of Medicine, 600

N Riverpoint Blvd, Spokane WA, 99202, U.S.A

Tel: $+1-360-591-6302$

Fax: +1-509-358-7882

E-mail: kyle.geiger@wsu.edu

Received: May 20, 2020

Revised: August 3, 2020

Accepted: August 25, 2020
Objective: There is a growing body of clinical evidence to support coccygectomy as an effective treatment for chronic coccygodynia. To date, the authors identified no other studies that describe and evaluate the paramedian surgical approach to coccygectomy and post-operative outcomes. Methods: A case report of 36 patients who underwent coccygectomy with a paramedian or midline approach. All patients had chronic coccydynia that was refractory to conservative treatment. Three patients were excluded from the study due to additional lumbar surgery (2) or missing data (1). Outcomes assessed were Visual Analog Scale (VAS), operative and post-operative complications, use of post-operative pain medication, and surgery time. Results: Included in the study were 24 females and 9 males with a mean age of 46 years (range 18-74 years). The most common etiologies were trauma (26) and idiopathic (7). Twenty seven patients underwent coccygectomy with a paramedian approach and 6 had the same procedure with the midline incision. There was a significantly lower infection rate in the paramedian group ( $p$-value $=0.00871)$. Conclusion: The paramedian and midline approach to coccygectomy are both viable treatments for coccygodynia. The two methods offer low complication rates and high patient satisfaction. Surgeon and patient preference should be taken into account when choosing a surgical approach for coccygectomy. Further examination comparing traditional midline vs. paramedian approach are needed to assess superiority.

Key Words: Chronic coccydynia, Coccygectomy, Paramedian approach, Surgical approach

\section{INTRODUCTION}

Coccygodynia is pain in the coccyx (tailbone area). Classic symptoms include midline pain located caudal to the sacrum and cephalad to the anus. This diagnosis is made clinically with pain in the coccyx region that is worse during activities such as sitting, standing, defecation, and sexual intercourse. Often, it is associated with lumbar pain, presumably from constant repositioning of the patient when sitting. Physical exam elicits tenderness to palpation of the coccyx. Clinical suspicion along with imaging techniques are used to make the diagnosis. Radiographs, especially sitting-versus-standing radiographs can be used to assess for dynamic instability. Common scans including Magnetic resonance imaging (MRI) and computerized tomography are often used to define the coccyx defect and to rule out other sources of sacrococcygeal pain ${ }^{1)}$. Female sex, Body Mass Index (BMI) and trauma have been established as independent prognostic factors for coccygodynia treatment ${ }^{2)}$. The higher incidence in women is perhaps due to differences in the shape and angles of the female pelvis and increased risk while giving birth" ${ }^{1)}$. Treatments include conservative therapies (physical therapy and capsaicin patch), interventional techniques (local injections with steroids and local anesthetic, pulsed radiofrequency ablation of ganglion, and extracorporeal shock wave therapy), and surgical techniques (complete and partial coccygectomies). There is a growing body of clinical evidence to support that coccygectomy is an effective treatment for patients with debilitating pain who had failed conservative therapies ${ }^{3-5}$. Failure is associated with certain pre-operative characteristics such as psychiatric illness, poor quality of life features, higher levels of pain, and use of opiates ${ }^{6}$. There is little research on the surgical approach to coccygectomy and its implications on post-operative outcomes. To our knowledge, this is the only study examining the paramedian approach to coccygectomy as compared to the midline approach.

\section{MATERIALS AND METHOD}




\section{Patients}

The investigation included a retrospective review of the electronic health care record of 36 patients from 2010-2019 with a history of coccygectomy for chronic coccygodynia performed by the most senior author. Patients were excluded if they underwent coccygectomy in addition to another procedure (2) such laminectomy or discectomy, did not trial conservative treatment, or if complete information could not be obtained from the medical record (1). The diagnosis of coccygodynia was confirmed based on a history of pain in the sacrococcygeal area exacerbated by sitting and recreated by palpation of the coccyx. Anteroposterior and lateral radiographs of the coccyx were obtained to aid clinical diagnosis. Patients were evaluated and deemed candidates for surgical treatment. Coccygectomy was preformed using a midline or novel paramedian approach described below. Recoded data included demographics, duration of symptoms, etiology, comorbidities, previous treatment, surgery time, complications, pre and post-operative 10-point visual analog scale (VAS) for pain, use of opiate drugs, and surgical approach. Data was transferred to a Microsoft Excel document and analyzed.

\section{Statistical Analysis}

It was preformed using a standard one-tailed $T$-test with alpha level set at $0.05(5 \%)$.

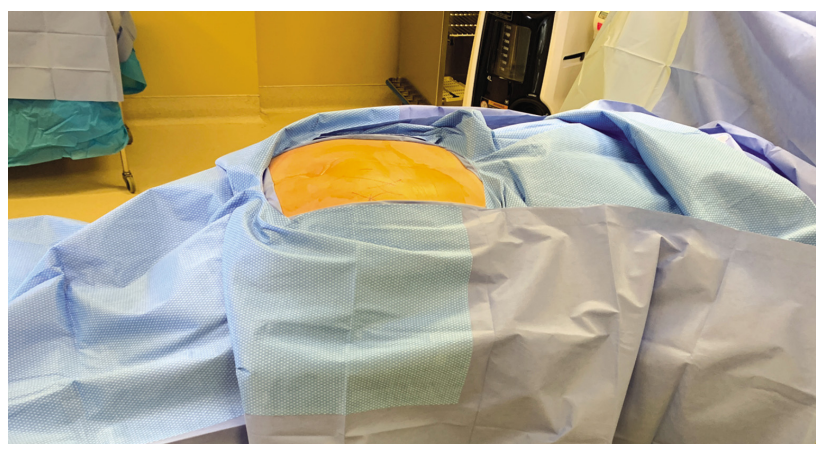

Fig. 1. Patient positioning and draping.

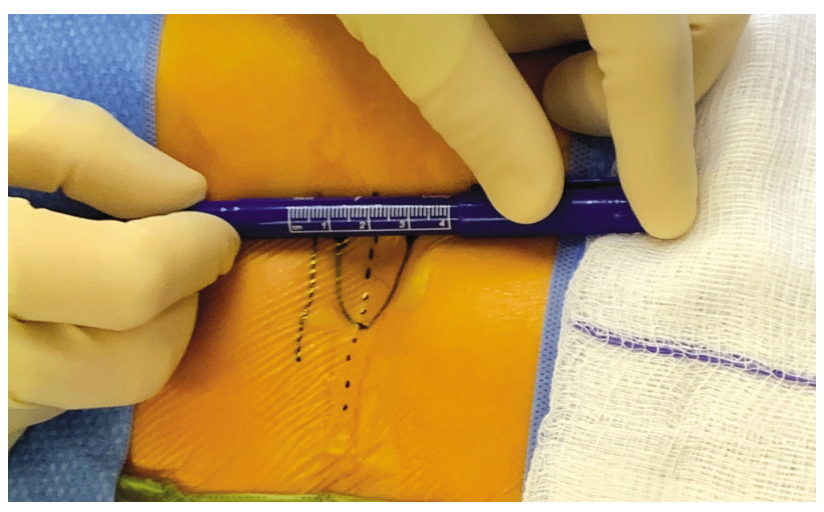

Fig. 2. Incision marked with pen.

\section{Description of the Paramedian Approach}

After operative consent is obtained, the patient is taken to the operating room to receive general anesthesia. The patient is then turned to the prone position on a Wilson ${ }^{\mathrm{TM}}$ frame mounted upon it. All pressure points are padded. The buttock and coccyx area are pre-prepped with isopropyl alcohol and allowed to dry. DuraPrep ${ }^{\mathrm{TM}}$ is then applied. The back and the coccyx are then sterilely draped utilizing loban ${ }^{\mathrm{TM}}$ as a final barrier draping (Fig. 1). After the surgical timeout is conducted, a $\sim 3 \mathrm{~cm}$ left paramedian incision is made adjacent to the intergluteal cleft approximately $2-3 \mathrm{~cm}$ cephalad to the anus (Figs. $2 \& 3$ ). A headlight is utilized for better visualization. The dissection is then taken tangentially to the midline of the coccyx in the sub-periosteal plane so as to obtain direct access dorsally (Figs. 4 \& 5). The coccyx is palpated along the way. Assurances are made to prevent interference with the rectum or the anal area. The edges of the coccyx are then trimmed off utilizing a 4 Kerrison, curved curets and coagulation electrocautery at a setting of 20 watts. The coccyx is removed (Fig. 6). The wound is then irrigated and anesthetized with 0.5 Marcaine in the absence of epinephrine, and without penetrating anterior to the surgical field at all. \#0 Vicryl is then utilized to close soft tissue to itself and to the residual elements of the sacrum using transosseous suture fixation (Fig. 7). The subcutaneous tissue is closed with 2-0 nylon using interrupted horizontal mattress sutures

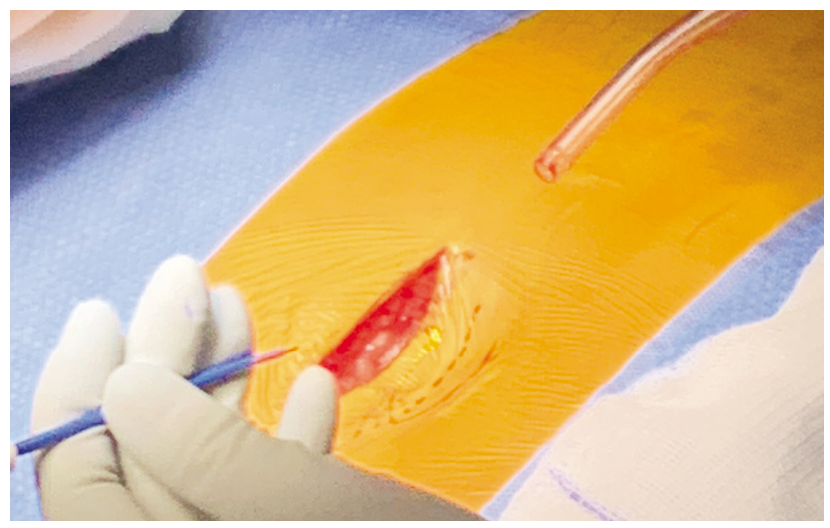

Fig. 3. Incision made laterally to intergluteal cleft.

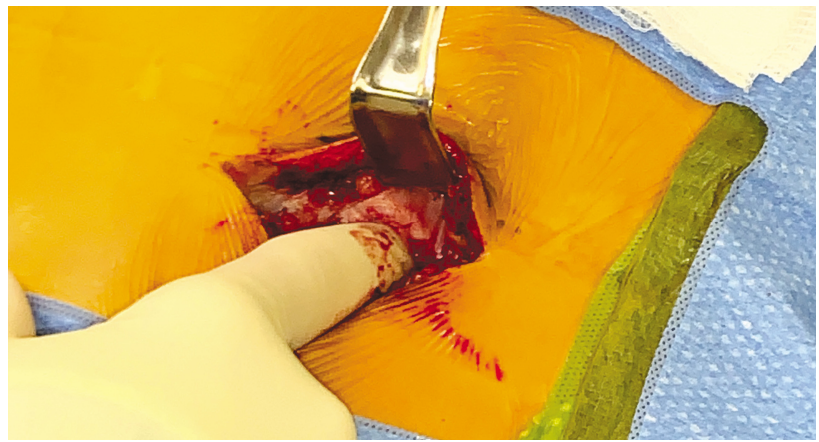

Fig. 4. Dissection and approach to periosteal plane. 
(Fig. 8). Sterile dressings are applied, and the patient is allowed to shower the wound without dressings on post-operative day number 2, after which, dressings are re-applied.

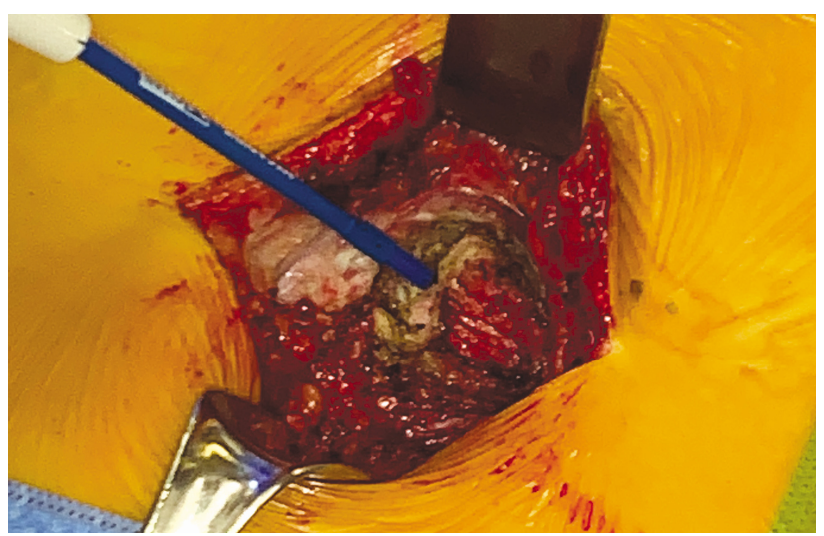

Fig. 5. Dissection down to coccyx.

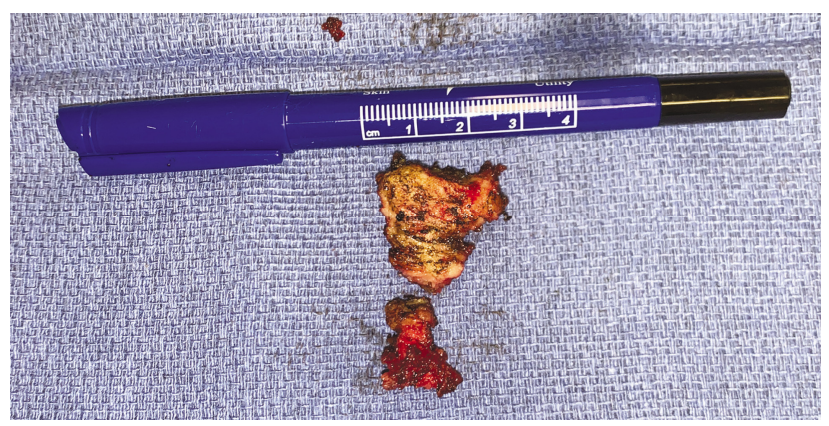

Fig. 6. Coccyx removed.

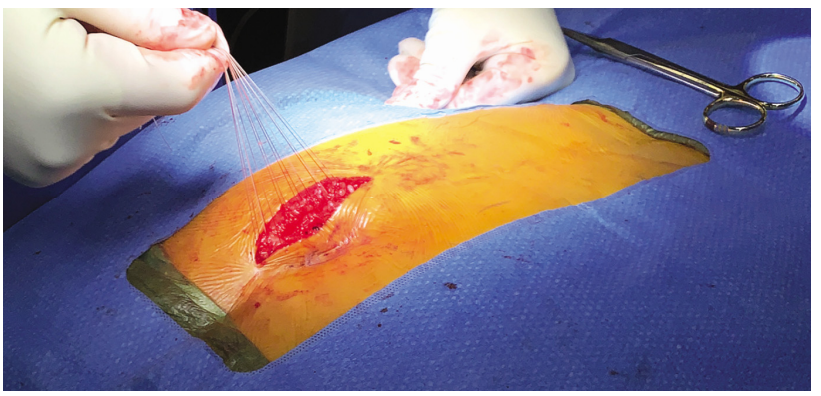

Fig. 7. Soft tissue fixation.

\section{Description of Midline Approach}

The midline approach, an earlier approach used by the senior author, is the same as the paramedian approach except that the initial incision is taken in the midline through the skin and subcutaneous tissue through the periosteum and then into the periosteal plane around the coccyx. The closure sequence is the same, but the amount of tissue closure is less because it is more direct.

\section{RESULTS}

Between 2010 and 2019, 36 patients with coccydynia (ICD-10 Code M53.3) with record of coccygectomy were identified. Three patients were excluded from the study due to additional spinal surgery (2) or missing data (1). Included in the study were 24 females and 9 males with a mean age of 46 years (range 18-74 years). The most common etiology was trauma (26) and idiopathic (7). 27 patients underwent coccygectomy with a paramedian approach and six had the same procedure with the midline approach. Demographics are displayed in Table 1. The average BMI was 27.8 (range 21.4-38.2). Injections, offloading,

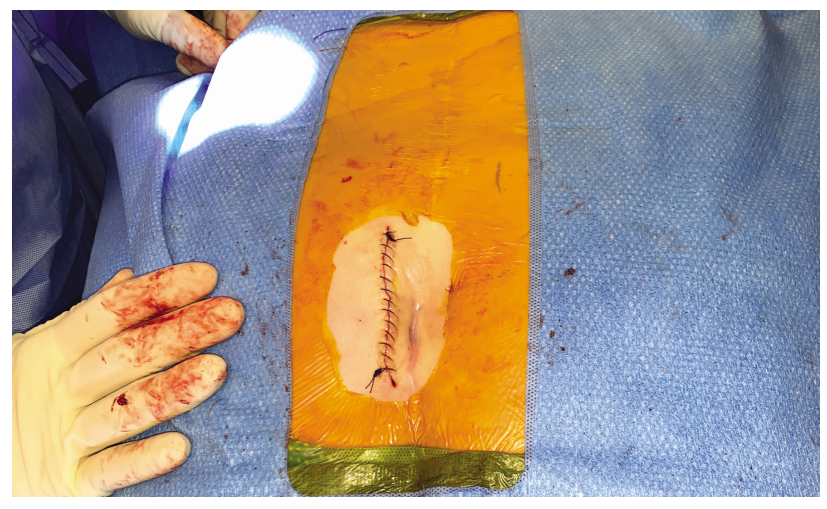

Fig. 8. Final closure of superficial and dermal layers.

Table 1. Patient demographics

\begin{tabular}{lll}
\hline \hline Age & 46 years & SD 15.4 \\
Sex & 9 Male & 24 Female \\
Etiology & 7 Idiopathic & 26 Trauma \\
Approach & 6 Midline & 27 Paracentral \\
\hline
\end{tabular}

Table 2. Comparison of wound complications by approach

\begin{tabular}{|c|c|c|c|}
\hline & Paracentral & Midline & \\
\hline$n$ & 27 & 6 & \\
\hline Wound complications & 3 & 4 & $\begin{array}{ll}\text { p-value } 0.000871 \\
\text { t-value } 3.42672\end{array}$ \\
\hline Intraoperative complications & 0 & $1^{*}$ & \\
\hline Patients requiring incision and drainage & 0 & 2 & \\
\hline
\end{tabular}

*Wound vacuum placed at end of operation. 
physical therapy, and non-steroidal anti-inflammatory medications were common conservative treatments reported in our patients. The average duration of symptoms was 8.2 years (range 2 months to 35 years). 25 of the patients required post-operative opioid pain medication, most commonly oxycodone $5-10 \mathrm{mg}$. For the paramedian group, 3 of 27 patients required antibiotics due to post-op infection. For the midline group 4 of 6 had wound complications requiring either incision and drainage and/or antibiotics. There was a significantly lower infection rate in the paramedian group $(\mathrm{p}=0.00871$, alpha $<0.05)$. No operative complications were noted in the paramedian group. In the midline group one patient required a wound-vacuum placed at the end of the operation. Data for wound complications is displayed in Table 2.

For the paramedian group 17 Patients reported a decrease in pain per VAS, 5 reported no change, 5 reported an increase in pain, and one reported complete resolution with a VAS score of 0 post-operatively. VAS data for the paramedian approach averaged 7.1 pre-operatively and 4.4 post operatively. These values were obtained at pre and post-op visits. On average, VAS scale was administered 7 days before surgery range (1-18 days) and 175 days after surgery range (17-529 days). The average surgery time in this group was 40.6 minutes. The maximun surgery time was 53 minutes and the minimum surgery time was 29 minutes. Surgery time was not recorded for 4 patients and those were excluded from the operating time analysis.

\section{DISCUSSION}

Coccygectomy for chronic coccydynia has been established as a promising treatment for pain refractory to conservative management ${ }^{3-6)}$. The most common complications after coccygectomy are infection and postoperative wound dehiscence. In a review of the literature Kleimeyer and colleagues report postoperative dehiscence and infection rates ranging from $0 \%$ to $30 \%{ }^{3)}$. Infection is most commonly with S. aureus ${ }^{7}$, however recent authors have reported using preoperative rectal enemas and broadening antibiotics to cover gram negative microbes ${ }^{8)}$. In our patients we use cefazolin intravenously with $1 \mathrm{gram}$ applied directly into the wound at the end of the case. Notably, we saw significantly less infections and/or postoperative dehiscence with the paramedian approach $(\mathrm{p}=0.000871)$.

We believe that the lower infection rate is due to the paramedian surgical approach in addition to antibiotic coverage and surgical technique that allows a deeper skin flap away from the less exposed intergluteal cleft. In the classic midline approach described by Key et al. ${ }^{9)}$ a two and one-half inch long midline vertical incision is made over the sacrococcygeal articulation and carried down to the bone. This approach described in 1937 is still the most commonly used approach today ${ }^{10-13)}$. We presume that the wound location within the intergluteal cleft acts as a nidus for infection. Our approach avoids this natural crevice and instead allows wound closure adjacent to intergluteal cleft. We also speculate that the location of the incision site may avoid direct pressure while sitting. Another small study of 25 patients by Bilgic et al. ${ }^{14)}$ suggests that periosteal preservation and closure are related to low risk of infection. They report an infection rate of $0 \%(0 / 14)$ with periosteal preservation vs $36 \%(4 / 11)$ without. However, the superficial surgical approach was not examined.

The paramedian approach does not appear to negatively impact surgery time. Our average surgery time was 40.6 minutes; 10 minutes lower than reported in a recent study with total excision of the coccyx ${ }^{15)}$.

Although our study involves a small sample size, the demographics are similar to recent studies ${ }^{111}$. Trauma remains the most common etiology, and middle age females also make up the majority of our patients. Limitations of this study include small sample size and lack of data relating to outcomes. Only one pain scale (VAS) was used to evaluate post-operative pain, while other studies employ multiple. The Coccygodynia Disability Index (CDI) which is a four-question instrument using questions 1 , 5,8 , and 9 from the Oswestry Disability Index ${ }^{16)}$, should be included for further studies of this approach. Another limitation was the small comparison group for the midline coccygectomy. There was a lack of available data in the midline group in regard to VAS reporting and surgery time.

\section{CONCLUSION}

The paramedian and midline approach to coccygectomy are both viable treatments for coccygodynia. While the traditional midline approach has low complication rates and high patient satisfaction, it is possible the paramedian approach offers these same positive outcomes. Surgeon and patient preference should be taken into account when choosing a surgical approach for coccygectomy. Further examination of the paramedian approach and its role in reducing infection is needed. Additional prospective studies comparing traditional midline vs. paramedian approach are needed to assess whether there is superiority of one method versus another.

Conflicts of Interest and Source of Funding: The authors have no direct financial relationships relevant to this article.

Funding: This study received no financial support or sponsorship.

\section{REFERENCES}

1. Foye PM. Coccydynia: Tailbone Pain. Phys Med Rehabil Clin N Am 28:539-549, 2017

2. Kodumuri P, Raghuvanshi S, Bommireddy R, Klezl Z: Coccydynia - Could age, trauma and body mass index be independent prognostic factors for outcomes of intervention? Ann R Coll Surg Engl 100:12-15, 2018

3. Kleimeyer JP, Wood KB, Lonne G, et al: Surgery for refractory coccygodynia: Operative versus nonoperative treatment. Spine 42:1214-1219, 2017

4. Elkhashab Y, Ng A: A review of current treatment options for coccygodynia. Curr Pain Headache Rep 22:28, 2018 
5. Sarmast AH, Kirmani AR, Bhat AR: Coccygectomy for coccygodynia: A single center experience over 5 years. Asian J Neurosurg 13:277-282, 2018

6. Hanley EN, Ode G, Jackson Iii BJ, Seymour R: Coccygectomy for patients with chronic coccydynia: A prospective, observational study of 98 patients. Bone Joint J 98-B:526-533, 2016

7. Doursounian L, Maigne J-Y, Faure F, Chatellier G: Coccygectomy for instability of the coccyx. Int Orthop 28:176-179, 2004

8. Doursounian L, Maigne J-Y, Cherrier B, Pacanowski J: Prevention of post-coccygectomy infection in a series of 136 coccygectomies. Int Orthop 35:877-881, 2011

9. Key A: Operative treatment of coccygodynia. J Bone Joint Surg 19:759-764, 1937

10. Aggarwal A, Kumar S, Kumar D: Factors influencing the evaluation and management outcomes of coccygodynia: A literature review. J Back Musculoskelet Rehabil 26:105-115, 2013

11. Karadimas EJ, Trypsiannis G, Giannoudis PV: Surgical treat- ment of coccygodynia: an analytic review of the literature. Eur Spine J 20:698-705, 2011

12. Sehirlioglu A, Ozturk C, Oguz E, Emre T, Bek D, Altinmakas M: Coccygectomy in the surgical treatment of traumatic coccygodynia. Injury 38:182-187, 2007

13. Ramieri A, Domenicucci M, Cellocco P, Miscusi M, Costanzo G: Acute traumatic instability of the coccyx: Results in 28 consecutive coccygectomies. Eur Spine J 22(Suppl 6):S939-944, 2013

14. Bilgic S, Kurklu M, Yurttaş Y, Ozkan H, Oguz E, Sehirlioglu A: Coccygectomy with or without periosteal resection. Int Orthop 34:537-541, 2010

15. Ogur HU, Seyfettinoğlu F, Tuhanioğlu Ü, Cicek H, Zohre S: An evaluation of two different methods of coccygectomy in patients with traumatic coccydynia. J Pain Res 10:881-886, 2017

16. Fairbank JC, Couper J, Davies JB, O'Brien JP: The Oswestry low back pain disability questionnaire. Physiotherapy 66:271273, 1980 